\title{
Globale Standards für klinische Studien nützen allen
}

\section{Thomas Cueni}

Generalsekretär/Geschäftsführer interpharma, Verband der forschenden pharmazeutischen Firmen der Schweiz

In der letzten SÄZ-Ausgabe plädierte Jacques Schiltknecht für mehr Verantwortung und Kontrolle der Pharmaunternehmen im Bereich der klinischen Forschung [1]. Das gemäss seiner Schilderung scheinbar auch in Ärztekreisen weitverbreitete Nicht-Wissen über den regulatorischen Rahmen für die klinische Forschung in der Schweiz und im Ausland ist bedauerlich und dürfte mit ein Grund dafür sein, dass Pharmafirmen immer wieder für die Durchführung klinischer Studien in Entwicklungs- und Schwellenländern kritisiert werden. So hat im September die Erklärung von Bern (EvB) behauptet, dass die Pharmaindustrie bei klinischen Versuchen in Entwicklungsländern «häufig gegen ethische Mindeststandards» verstosse und Studien aus Kostengründen immer öfter in Entwicklungsländer auslagere.

\section{Die Pharmaunternehmen haben ein ureigenes Interesse daran, dass überall die GCP-Standards tatsächlich eingehalten werden.}

Korrespondenz:

Thomas B. Cuen interpharma iph Petersgraben 35 CH-4003 Basel Tel. 0612643417
Wie Schiltknecht aber richtig schreibt, gibt es für die Zunahme klinischer Studien in Schwellen- und teils Entwicklungsländern eine Reihe von Gründen: medizinische (verschiedene ethnische Populationen), regulatorische (obligatorisch für die Zulassung in gewissen Ländern), die Prävalenz von bestimmten Krankheiten, tiefere Kosten. Letzteres spielt sicher auch eine Rolle, ist aber nur relevant, weil die Infrastruktur in manchen Ländern deutlich verbessert worden ist. Dennoch ist es nach wie vor so, dass der Grossteil der meist länderübergreifenden Studien, die für die Zulassung in der Schweiz bei Swissmedic eingereicht werden, in den traditionellen Industrieländern durchgeführt wird. Es gibt von Swissmedic zwar keine offiziellen Zahlen dazu. Da die Zulassungsdossiers in der Schweiz aber konsistent mit den in der EU eingereichten Unterlagen sind, dürfte die Verteilung ähnlich sein wie bei der europäischen $\mathrm{Zu}$ lassungsbehörde EMA: Über 73\% aller Teilnehmer von klinischen Studien, die zwischen 2005 und 2010 bei der EMA im Rahmen der zentralen Zulassungsverfahren eingereicht wurden, stammten aus den EU/EWR/EFTA-Ländern sowie aus Nordamerika. 8,7\% aller Teilnehmenden kamen aus den asiatisch- pazifischen Ländern sowie dem Nahen Osten, der Anteil der Studienteilnehmer aus Zentral- und Südamerika belief sich auf 8,5\%. Weniger als 3\% aller Studienteilnehmer stammten aus Afrika [2]. Es kann also keine Rede davon sein, dass «etwa die Hälfte der klinischen Versuche in Ländern vorgenommen wird, in welchen grosse Armut herrscht und/oder Menschenrechte missachtet werden». Die Behauptung, dass «häufig gegen ethische Mindeststandards» verstossen werde, ist im Übrigen ein Pauschalvorwurf, der den Behörden in Nicht-OECDLändern per se unterstellt, dass sie nicht vertrauenswürdig seien und zudem übersieht, dass es global gültige Standards gibt. Wenn es Verstösse gibt - was ja auch schon in Industrieländern vorgekommen sein soll - müssen sie geahndet werden. Das steht ausser Frage, und nicht nur die betroffenen Studienteilnehmer, sondern auch Pharmaunternehmen, deren Reputation auf dem Spiel steht, haben ein Interesse daran. Das gilt auch, wie der Fall VanTx zeigte, der in der Schweiz vor 15 Jahren hohe Wellen warf, bei der Durchführung klinischer Studien mit externen Partnern (CROs). Mangelnde Sorgfaltspflicht und Aufsicht gegenüber CROs ist für Pharmafirmen nicht nur ein Reputationsrisiko, sondern kann auch teure Folgen haben.

Wie Schiltknecht richtig schreibt, gelten die Standards der Good Clinical Practice (GCP), die auf der Deklaration von Helsinki des Weltärztebundes basieren, weltweit, d.h. auch in Entwicklungsländern. So muss in jedem Fall eine Einwilligung der Teilnehmer vorliegen (informierte Zustimmung), es darf keinen Teilnahmezwang geben, Nutzen und Risiko müssen für die Teilnehmenden in einem günstigen Verhältnis stehen, und vor jeder Studie braucht es zwingend eine unabhängige Überprüfung einer Ethikkommission. Darüber hinaus müssen zahlreiche weitere Punkte erfüllt sein. Dazu kommen weitere international anerkannte Regelwerke und ethische Selbstverpflichtungen der forschenden Pharmaindustrie, auf europäischer Ebene vom Europäischen Pharmaverband EFPIA [3], in der Schweiz - wo die GCP-Richtlinien ohnehin gesetzlich gelten - ist die Verpflichtung auf die Einhaltung der GCP-Kriterien im Pharmakodex [4] geregelt.

Damit sich klinische Studien gemäss GCP durchführen lassen, ist ein Mindestmass an Struktur und Infrastruktur nötig. Ist dies nicht der Fall, werden die Studien von den Arzneimittelbehörden für die Zulas- 
sung nicht anerkannt. In der Schweiz ist in der Arzneimittel-Zulassungsverordnung (AZMV) geregelt, dass die Dokumentation über die klinischen Prüfungen belegen muss, dass die Untersuchungen am Menschen nach den anerkannten GCP-Regeln durchgeführt worden sind. Die Pharmaunternehmen haben also ein ureigenes Interesse daran, dass überall, wo klinische Versuche durchgeführt werden, die GCP-Standards auch tatsächlich eingehalten werden. Auch in der EU gilt eine ähnliche «equivalence rule» [5], derzufolge klinische Versuche, die Basis einer Zulassung bei der EMA sind, nach gleichen Standards (also GCP) durchgeführt werden müssen wie in der EU. Andernfalls werden auch sie nicht anerkannt. Weiter müssen die Unternehmen die klinischen Versuche intern streng überwachen.

\section{Über $73 \%$ aller Teilnehmer an klinischen Studien zwischen 2005 und 2010 stammten aus den EU/EWR/ EFTA-Ländern oder Nordamerika.}

Wie Schiltknecht richtig bemerkt, sind einige seiner als Diskussionsanstoss erwähnten Punkte bereits in firmeneigenen Verpflichtungen und diversen internationalen Kodices enthalten. Dies gilt namentlich für die Registrierung aller klinischen Studien, wobei die meisten globalen Firmen das amerikanische Web-Portal clinicaltrials.gov des National Institute of Health (NIH) nutzen. Und selbstverständlich werden die Daten den zuständigen Arzneimittelbehörden vollständig und transparent übermittelt. Erst jüngst haben sich die europäischen und amerikanischen Pharmaverbände auf Grundsätze für mehr Transparenz über klinische Studien geeinigt. Dazu gehört nicht nur die Veröffentlichung einer Zusammenfassung klinischer Studienreports, sondern auch die Bereitschaft, die Daten (auf anonymisierter Patientenbasis) für begründete Forschungsprojekte unabhängiger Forscher zur Verfügung zu stellen.

Schliesslich gehen viele Pharmaunternehmen mit eigenen Richtlinien noch weiter als die GCP. So haben sich gerade auch die grossen Schweizer Pharmafirmen Roche und Novartis dazu verpflichtet, klinische Versuche nur in Ländern durchzuführen, in denen die getesteten Medikamente - sofern sie sich als sicher und wirksam erweisen - auf den Markt gebracht werden. Bei überlebenswichtigen Medikamen- ten wird zudem der Zugang der Studienteilnehmenden über die Studie hinaus bis zur Vermarktung des Präparats garantiert [6]. Kurzum: Der medizinische Fortschritt, der dank der Forschung in diesen Ländern generiert wird, kommt also auch direkt der lokalen Bevölkerung zugute. Somit wäre der Verzicht auf klinische Versuche in ärmeren Ländern auch ethisch fragwürdig. Zudem tragen die westlichen Pharmaunternehmen zum Transfer von Wissen und Know-how in diese Länder bei, was zu einer besseren Gesundheitsversorgung führt und wiederum der Lokalbevölkerung nützt.

Die Behauptung der EvB, die Globalisierung klinischer Forschung geschehe einzig aus Kostengründen und füge der Lokalbevölkerung mehr Schaden als Nutzen zu, ist schlicht falsch. Dank der weltweiten Umsetzung von GCP kommt es zu einer Winwin-win-Situation, von der sowohl die lokale Bevölkerung, die Bevölkerung in anderen Ländern wie auch die Pharmaindustrie profitieren. Sicherlich gibt es dabei noch Potential für weitere Verbesserungen.

\section{Literatur}

1 Schiltknecht J. Fair Pharma? Klinische Versuche: Verantwortung und Kontrolle. Schweiz Ärztezeitung. 2013;94(50):1922-4.

2 Reflection paper on ethical and GCP aspects of clinical trials of medicinal products for human use conducted outside of the EU/EEA and submitted in marketing authorisation applications to the EU Regulatory Authorities, April 2012 (www.ema.europa.eu/docs/ en_GB/document_library/Regulatory_and_ procedural_guideline/2012/04/WC500125437.pdf).

3 z. B. EFPIA Position paper: Clinical trial standards in developing and emerging countries in the context of the proposed EU Regulation on clinical trials on medicinal products for human use, October 2012 (http://www.efpia.eu/uploads/Modules/Documents/ efpia_position_paper_key_messages_third_countries_ d1tc_-_30.10.2012.pdf)

4 www.scienceindustries.ch/engagements/ pharmakodex

5 European Clinical Trials Directive 2001/20/EC; European Directive 2003/63/EC Annex 1 amending Directive 2001/20/EC.

6 Novartis:

Clinical Trials in Developing Countries, www.novartis. com/downloads/corporate-responsibility/resources/ positions/clinical-trials-developing-markets.pdf; Roche:

Roche Clinical Trials Policy in Low and Middle Income Developing Countries, www.roche.com/clinical_trials_policy_in_low_and_middle_income_developing countries.pdf 